\title{
Clinical Characteristics and Outcomes in Immune Checkpoint Inhibitor Therapy-Associated Myocarditis
}

\author{
Ravi A. Thakker ${ }^{\mathrm{a}, \mathrm{h}}{ }^{\circ}$, Marissa A. Lee ${ }^{\mathrm{a}}$, Aiham Albaeni ${ }^{\mathrm{b}}$, Ayman Elbadawi ${ }^{\mathrm{c}}$, Krishna H. Suthar ${ }^{\mathrm{d}}$, \\ Christopher Perez ${ }^{\mathrm{e}}$, Lindsay K. Sonstein ${ }^{\mathrm{a}}$, Norman M. Farr ${ }^{\mathrm{a}}$, Rohit Venkatesan ${ }^{\mathrm{f}}$, \\ Wissam Khalife $^{b}$, Rafic F. Berbarie ${ }^{g}$, Khaled F. Chatila ${ }^{b}$
}

\begin{abstract}
Immune checkpoint inhibitor (ICI) therapy has played an important role in the treatment of several groups of cancers. Although a life prolonging treatment, many side effects have been shown with ICI therapy. This study looked at individual level clinical characteristics and outcomes with ICI therapy in patients who developed ICI-related myocarditis. A comprehensive review of the National Library of Medicine PubMed database was performed. Inclusion criteria were all studies that were composed of case reports and case series of individual patients undergoing ICI therapy that developed myocarditis. To appreciate individual patient level data, observational studies, clinical trials, systematic reviews, and meta-analyses were excluded. Our search yielded 333 results with 71 cases reviewed of ICI therapy-related myocarditis. The findings included an average age of 68 years, higher incidence in men, and pretreatment cardiac history of hypertension. Melanoma was the most prevalent malignancy with nivolumab being the most used ICI therapy. Heart failure was the most prevalent adverse event that was co-prevalent with myocarditis. Corticosteroid therapy alone was the most utilized therapy to treat ICI-related myocarditis. Mortality was seen in nearly half of the patient population. Our study reviewed the preexisting literature of prior reported myocarditis secondary to ICI therapy. Periodic surveillance should be performed by the cardio-oncologist and internist. Due to the expanding role of ICI therapy in treating a variety of cancer pa-
\end{abstract}

Manuscript submitted August 31, 2021, accepted September 13, 2021

Published online September 29, 2021

${ }^{a}$ Department of Internal Medicine, University of Texas Medical Branch, Galveston, TX, USA

${ }^{b}$ Division of Cardiology, University of Texas Medical Branch, Galveston, TX, USA

'Section of Cardiology, Baylor College of Medicine, Houston, TX, USA

${ }^{\mathrm{d}}$ Department of Hematology and Medical Oncology, Baylor Scott and White Medical Center, Temple, TX, USA

${ }^{e}$ Cardiovascular Institute, Baylor Scott and White Medical Center, Temple, TX, USA

fDepartment of General Oncology, The University of Texas MD Anderson Cancer Center, Galveston, TX, USA

gDivision of Cardiology, UT Southwestern Medical Center, Dallas, TX, USA

${ }^{\mathrm{h}}$ Corresponding Author: Ravi A. Thakker, Department of Internal Medicine, University of Texas Medical Branch, Galveston, TX, USA.

Email: ravi.a.thakker@gmail.com

doi: https://doi.org/10.14740/cr1319 tients, appreciation of its impact on the development of myocarditis is needed.

Keywords: Immune checkpoint inhibitor; Myocarditis; Heart failure; Cardio-oncology

\section{Introduction}

Immune checkpoint inhibitor (ICI) therapy has played an important role in the treatment of several groups of cancers. Through blockade of programmed cell death protein 1 (PD1), programmed death-ligand 1 (PD-L1), and cytotoxic T-lymphocyte-associated protein 4 (CTLA-4) immune checkpoints, this class of immunotherapies has been shown to even provide long-term remission [1]. Currently, seven ICI therapies exist that have been approved by the US Food and Drug Administration. These seven therapies treat a wide variety of malignancies and include ipilimumab approved in 2011, nivolumab approved in 2014, pembrolizumab approved in 2014, cemiplimab approved in 2018, avelumab approved in 2017, atezolizumab approved in 2016, and durvalumab approved in 2017. Although immune check point inhibitors have improved survival in many cancer patients, many side effects have been appreciated. In particular, myocarditis has been shown to be one of the deadliest. The mortality rate of ICI-associated myocarditis has been described as up to $50 \%$ with an incidence reaching an average of $0.5 \%$ [2]. This study looked at individual level clinical characteristics and outcomes with ICI therapy in patients who developed ICI-related myocarditis.

\section{Methods}

A comprehensive review of the National Library of Medicine PubMed database was performed. Keywords included in the search were "immune checkpoint inhibitor" and "myocarditis." Database results were confirmed by multiple authors (RAT and MAL). Inclusion criteria were all studies that were composed of case reports and case series of individual patients undergoing ICI therapy that developed myocarditis to assess individual patient level data. Exclusion criteria for this review were case reports and case series where myocarditis was 


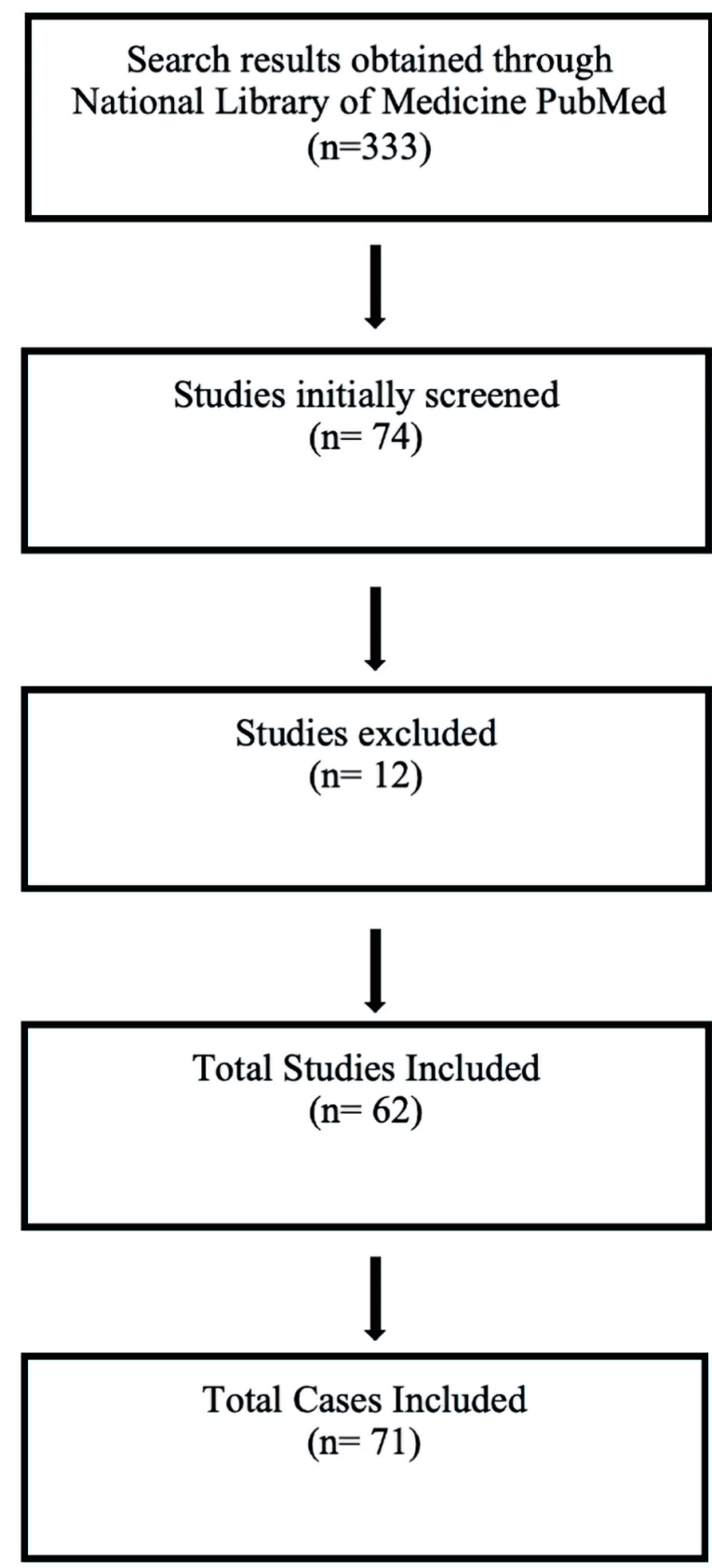

Figure 1. Case selection.

not one of the main immune therapy-related adverse events. Observational studies, clinical trials, systematic reviews, and meta-analyses were excluded due to difficulty assessing individual patient level data. Systematic analyses were not performed due to variability in data.

\section{Results}

Our search yielded 333 results with 71 cases reviewed of ICI therapy-related myocarditis (Fig. 1). The average age of the
Table 1. Patient Characteristics

\begin{tabular}{|c|c|c|}
\hline & $\mathbf{N}$ & $\%$ \\
\hline \multicolumn{3}{|l|}{ Gender } \\
\hline Male & 46 & $64.8 \%$ \\
\hline Female & 24 & $33.8 \%$ \\
\hline No gender provided & 1 & $1.4 \%$ \\
\hline \multicolumn{3}{|l|}{ Pretreatment cardiovascular diagnosis } \\
\hline Atrial fibrillation/atrial flutter & 1 & $4.8 \%$ \\
\hline History of arrhythmia & 2 & $9.5 \%$ \\
\hline History of coronary artery bypass grafting & 2 & $9.5 \%$ \\
\hline History of valve repair & 1 & $4.8 \%$ \\
\hline Hyperlipidemia & 6 & $28.6 \%$ \\
\hline Hypertension & 19 & $90.5 \%$ \\
\hline Myocardial infarction & 1 & $4.8 \%$ \\
\hline Peripheral artery disease & 2 & $9.5 \%$ \\
\hline Not provided/not applicable & 50 & \\
\hline \multicolumn{3}{|l|}{ Malignancy } \\
\hline$>1$ malignancy & 2 & $2.8 \%$ \\
\hline Clear cell renal cell carcinoma (ccRCC) & 2 & $2.8 \%$ \\
\hline Chronic myelomonocytic leukemia (CMML) & 1 & $1.4 \%$ \\
\hline Glioblastoma & 1 & $1.4 \%$ \\
\hline Head and neck squamous cell carcinoma & 3 & $4.2 \%$ \\
\hline Hepatocellular carcinoma (HCC) & 1 & $1.4 \%$ \\
\hline Large cell neuroendocrine carcinoma (LCNEC) & 1 & $1.4 \%$ \\
\hline Lymphoma & 1 & $1.4 \%$ \\
\hline Melanoma & 24 & $33.8 \%$ \\
\hline Mesothelioma & 3 & $4.2 \%$ \\
\hline Non-small cell lung carcinoma (NSCLC) & 18 & $25.4 \%$ \\
\hline Periocular squamous cell carcinoma & 1 & $1.4 \%$ \\
\hline Prostate adenocarcinoma & 2 & $2.8 \%$ \\
\hline Renal cell carcinoma (RCC) & 5 & $7.0 \%$ \\
\hline Sarcoma-alveolar soft part & 1 & $1.4 \%$ \\
\hline Serous endometrial carcinoma & 1 & $1.4 \%$ \\
\hline Thymic cancer & 1 & $1.4 \%$ \\
\hline Thymoma & 1 & $1.4 \%$ \\
\hline Urothelial cancer & 2 & $2.8 \%$ \\
\hline
\end{tabular}

patient population was 68 years. Males accounted for most cases at $64.8 \%$, while females accounted for $33.8 \%$. Gender was not provided in one case. The most common pretreatment cardiovascular diagnosis that patients had was hypertension accounting for $90.5 \%$. Hyperlipidemia was the second most common cardiac comorbidity at $28.6 \%$. Prior cardiovascular diagnosis was either not reported or not applicable in 50 cases. The most common malignancy reported was melanoma at $33.8 \%$. Non-small cell lung carcinoma (NSCLC) accounted for the second most reported malignancy at $25.4 \%$ (Table 1). 
The most common ICI therapy reported was nivolumab accounting for $38 \%$ of cases. A combination of multiple ICI therapy was used in $28.2 \%$ of cases. The most common adverse event seen with co-prevalent myocarditis was heart failure in $38.5 \%$ of cases. The second most co-prevalent adverse event was heart block seen in $27.7 \%$ of cases. Adverse events were not reported in six cases. Treatment with corticosteroid therapy alone for ICI-associated myocarditis accounted for $56.3 \%$ of cases. Any combination of therapies including plasma exchange or plasmapheresis was seen in $11.3 \%$ of cases. Therapy was either not stated or unknown in two cases. Death was seen in $49.3 \%$ of cases. One case did not report mortality outcomes (Tables 2, 3) [3-64].

\section{Discussion}

Our study reviewed the preexisting literature of prior reported myocarditis secondary to ICI therapy. Although variability in data exists, individual patient level outcomes appreciated in our study were a higher incidence of ICI-associated myocarditis in males compared to females. This adverse effect was also seen mostly in patients around 68 years of age. Hypertension was the most common pretreatment cardiovascular diagnosis. Melanoma was the most prevalent malignancy, with nivolum$\mathrm{ab}$ being the most used ICI therapy. Other notable outcomes included heart failure being the most prevalent adverse event that was co-prevalent with myocarditis. Corticosteroid therapy alone, with either methylprednisolone or prednisone, was the most utilized therapy to treat ICI-related myocarditis. Mortality was seen in nearly half of the patient population. There was notable higher incidence of mortality among patients on nivolumab $3 \mathrm{mg} / \mathrm{kg}$ compared to $2 \mathrm{mg} / \mathrm{kg}$. One of the major limitations of our review was that in several of the cases prior medications, especially prior beta blocker use, were not mentioned [3-64].

\section{Pathophysiology}

Immunotherapy is a biological therapy, derived from living organisms that is used to treat cancer. While the immune system normally functions to destroy cancer cells, some cancers have found ways to avoid destruction [65]. The anti-PD-1 monoclonal antibodies $(\mathrm{mAb})$ include nivolumab, pembrolizumab, and cemiplimab. These mAbs bind to PD-1 and prevent binding with PD-L1 and programmed death-ligand 2 (PD-L2) on the tumor surface. This allows for PD-1-associated immune response to act against tumor cells [66]. Pembrolizumab and cemiplimab are also anti-PD-1 mAbs; however, their differences lie in the variable regions in which antigen binding occurs. This explains the difference in indications between the drugs, despite their nearly identical mechanism [67, 68]. Like the anti-PD-1 agents, the anti-PD-L1 antibodies block PD-L1 on the tumor surface; these anti-PD-L1 therapies include avelumab, atezolizumab, and durvalumab [69].

Ipilimumab, the first FDA-approved ICI, is a $\mathrm{mAb}$ that binds to CTLA- 4 and blocks interaction with its ligands CD80
(B7-1) and CD86 (B7-2). Through this inhibition of CTLA4 signaling, regulatory T-cell function is reduced, leading to increased T-cell and anti-tumor immune responses [70, 71]. Combination therapy with nivolumab (anti-PD-1 mAb) and ipilimumab (anti-CTLA-4 mAb) is currently used in many different cancers, leading to greater response rates and overall survival. On the contrary, these advances come with more immune-related adverse effects [72]. ICI-associated myocarditis has been noted as being a seemingly rare immune-related adverse effect in prior literature, although reporting of this adverse effect has grown over time [73].

Currently, there is no accepted mechanism for which this occurs; however, several suggested mechanisms exist. One proposed mechanism of ICI-associated myocarditis is through $\mathrm{T}$ cell infiltration. Johnson et al reported two cases of lethal myocarditis in combination with myositis in patients receiving nivolumab in combination with ipilimumab [74]. In their study, post-mortem cardiac histopathology showed T-cell infiltration with CD4 and CD8 positivity. It was also found that the patients shared high-frequency T-cell receptor sequences in the skeletal and cardiac muscle as well as tumor cells. Through these interactions, the authors propose that $\mathrm{T}$ cells target a shared antigen on the tumor, cardiac myocytes, and skeletal muscle which leads to cellular injury. Another similar mechanism discussed is T-cell receptors targeting tumor antigens and different yet similar muscle antigen. Finally, they propose that high-frequency T-cell receptors in both muscle and tumor cells deceive T-cells into targeting the wrong antigen [74].

In studies on mice, PDL-1 was found to be cardioprotective against T-cell-associated injury [74, 75], and mice deficient in the programmed cell death-1 immunoinhibitory coreceptor developed autoimmune dilated cardiomyopathy due to autoantibodies against troponin I [76]. CTLA-4, the target of ipilimumab, has been shown to have a role in T-cell activation. Tivol et al demonstrated that CTLA-4 deficient mice developed myocarditis leading to death within 3 - 4 weeks of life, showing the negative role and cardioprotective effect of CTLA-4 [77]. When CTLA-4 is used as an ICI, the antiCTLA-4 antibody interferes with CTLA-4 and B7-1, effectively lowering the activation threshold of cardiac reactive $\mathrm{T}$ cells [78]. Therefore, oncologic treatments using ICIs causing blockade of PD-1, PDL-1, and CTLA-4 have been implicated in promoting cardiac myocyte injury [79].

\section{Diagnostic considerations in ICI-associated myocarditis}

Diagnosis of ICI-related myocarditis involves obtaining a comprehensive cardiac profile. Review of systems will usually be positive for symptoms such as angina, dyspnea, orthopnea, and lower extremity edema. Physical examination findings may include development of a S3 gallop, jugular venous distention, lower extremity pitting edema, tachycardia, and hypotension with cardiogenic shock. Pertinent laboratory markers include obtaining troponin and brain natriuretic peptide (BNP) which may be abnormal. Electrocardiogram can also be helpful in assessing for arrhythmias, although at times may be normal [80]. The initial imaging modality should be echocardiogram with close assessment of global longitudinal strain and 
Table 2. Patient Outcomes

\begin{tabular}{|c|c|c|}
\hline & $\mathbf{N}$ & $\%$ \\
\hline \multicolumn{3}{|l|}{ ICI therapy } \\
\hline Atezolizumab & 1 & $1.4 \%$ \\
\hline Cemiplimab & 1 & $1.4 \%$ \\
\hline Durvalumab & 1 & $1.4 \%$ \\
\hline Ipilimumab & 1 & $1.4 \%$ \\
\hline Multiple immune checkpoint inhibitors used & 20 & $28.2 \%$ \\
\hline Pembrolizumab & 19 & $26.8 \%$ \\
\hline Sintilimab & 1 & $1.4 \%$ \\
\hline \multicolumn{3}{|l|}{ Adverse events with coprevalent myocarditis } \\
\hline Acute kidney injury & 6 & $9.2 \%$ \\
\hline Acute liver injury & 12 & $18.5 \%$ \\
\hline Autoimmune diabetes mellitus & 1 & $1.5 \%$ \\
\hline Colitis & 3 & $4.6 \%$ \\
\hline Encephalitis & 1 & $1.5 \%$ \\
\hline Heart block & 18 & $27.7 \%$ \\
\hline Heart failure & 25 & $38.5 \%$ \\
\hline Hypophysitis & 1 & $1.5 \%$ \\
\hline Myasthenia gravis/crisis & 14 & $21.5 \%$ \\
\hline Myositis & 16 & $24.6 \%$ \\
\hline Pericardial effusion & 1 & $1.5 \%$ \\
\hline Pneumonitis & 5 & $7.7 \%$ \\
\hline Anti-thymocyte globulin (ATG) alone & 0 & $0.0 \%$ \\
\hline Supportive care & 1 & $1.4 \%$ \\
\hline Corticosteroid + immunosuppressant (tacrolimus, mycophenolate mofetil) & 3 & $4.2 \%$ \\
\hline Corticosteroid + IVIG & 6 & $8.5 \%$ \\
\hline Corticosteroid + IVIG + immunosuppressant & 1 & $1.4 \%$ \\
\hline Not stated/no treatment & 2 & $2.8 \%$ \\
\hline Corticosteroid + ATG & 2 & $2.8 \%$ \\
\hline Corticosteroid + tofacitinib & 1 & $1.4 \%$ \\
\hline Corticosteroid + IVIG + tofacitinib & 1 & $1.4 \%$ \\
\hline Corticosteroid + infliximab & 3 & $4.2 \%$ \\
\hline Corticosteroid + IVIG + infliximab & 1 & $1.4 \%$ \\
\hline Corticosteroid + immunosuppressant + ATG + infliximab & 1 & $1.4 \%$ \\
\hline Any combination including plasma exchange/plasmapheresis & 8 & $11.3 \%$ \\
\hline \multicolumn{3}{|l|}{ Mortality } \\
\hline Alive & 35 & $49.3 \%$ \\
\hline Death & 35 & $49.3 \%$ \\
\hline Unknown & 1 & \\
\hline
\end{tabular}


Table 3. Associated Mortality

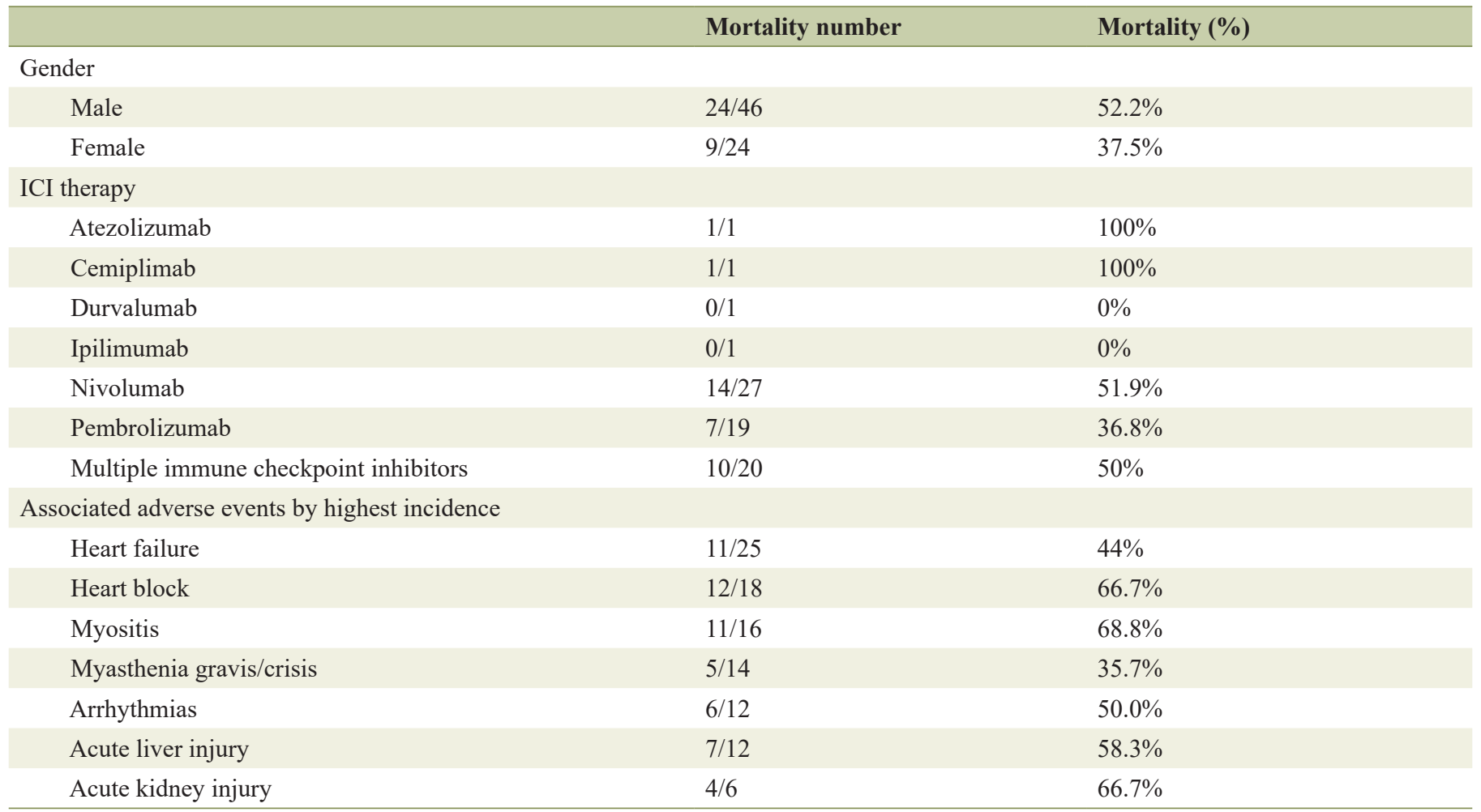

left ventricular ejection fraction. Cardiac magnetic resonance imaging has also been noted as being helpful in evaluation of the myocardium in ICI-associated myocarditis but with overall poor sensitivity. Ultimately, the main determining diagnostic modality is endomyocardial biopsy [81]. Endomyocardial biopsy usually demonstrates a predominance of lymphocytes with myocyte necrosis along with fibrosis and inflammation [82]. Global longitudinal strain has recently become an area of interest in assessing the degree of cardiac insult in patients who develop ICI-associated cardiotoxicity. In an international retrospective study, 101 cases of myocarditis in patients undergoing ICI therapy were evaluated. Notable findings from this large study were that global longitudinal strain was decreased in patients who developed myocarditis, with this decrease found in patients with both reduced and preserved ejection fractions. Furthermore, there was correlation between major adverse cardiovascular events in patients with low global longitudinal strain and myocarditis [83].

\section{Treatment modalities for ICI-associated myocarditis}

Although, no definitive treatment protocols exist, guidelines for the treatment of ICI-related myocarditis have been developed by the American Society of Clinical Oncology. For patients presenting with grade 1 chemotoxicity, which is elevation of biomarkers in the absence of symptoms or higher, ICI therapy should be stopped and discontinued permanently. High-dose prednisone at $1-2 \mathrm{mg} / \mathrm{kg}$ should be administered promptly.
Patients who do not respond to high-dose prednisone should be trialed on methylprednisolone $1 \mathrm{~g}$ every day in combination with immunosuppressive therapy such as mycophenolate mofetil, infliximab, or antithymocyte globulin until resolution of myocarditis [84]. In patients with New York Heart Association class III or IV heart failure, the use of infliximab is relatively contraindicated. For this subclass of patients with heart failure and ICI-related myocarditis, the use of antithymocyte globulin or tacrolimus in combination with high-dose steroids has been shown to be more appropriate [85].

\section{Conclusions}

As demonstrated in our review, the understanding of the mechanisms underlying ICI-associated myocarditis is still ongoing. Furthermore, patients may present asymptomatically or in cardiogenic shock. Prior to initiation of therapy, a baseline cardiac profile including biomarkers such as troponin and BNP should be obtained in conjunction with a baseline echocardiogram. Periodic surveillance should be performed by the cardio-oncologist and internist. Due to the expanding role of ICI therapy in treating a variety of cancer patients, further appreciation of its impact on the development of myocarditis is needed.

\section{Acknowledgments}

None to declare. 


\section{Financial Disclosure}

None to declare.

\section{Conflict of Interest}

The authors declare that they do not have a conflict of interest.

\section{Author Contributions}

All authors had access to the data and a role in writing the manuscript. RAT: conceptualization, data curation, writing of draft preparation, reviewing, and editing. MAL: data curation, writing of draft preparation, reviewing, and editing. AA, AE, KHS, and CP: writing of reviewing and editing. LKS, NMF, $\mathrm{RV}, \mathrm{WK}, \mathrm{RFB}$, and KFC: writing of reviewing and editing, expert opinion.

\section{Data Availability}

The authors declare that data supporting the findings of this study are available within the article.

\section{References}

1. Robert C. A decade of immune-checkpoint inhibitors in cancer therapy. Nat Commun. 2020;11(1):3801.

2. Palaskas N, Lopez-Mattei J, Durand JB, Iliescu C, Deswal A. Immune checkpoint inhibitor myocarditis: pathophysiological characteristics, diagnosis, and treatment. J Am Heart Assoc. 2020;9(2):e013757.

3. Imai R, Ono M, Nishimura N, Suzuki K, Komiyama N, Tamura T. Fulminant myocarditis caused by an immune checkpoint inhibitor: a case report with pathologic findings. J Thorac Oncol. 2019;14(2):e36-e38.

4. Mahmood SS, Chen CL, Shapnik N, Krishnan U, Singh HS, Makker V. Myocarditis with tremelimumab plus durvalumab combination therapy for endometrial cancer: A case report. Gynecol Oncol Rep. 2018;25:74-77.

5. Yamaguchi S, Morimoto R, Okumura T, Yamashita Y, Haga T, Kuwayama T, Yokoi T, et al. Late-onset fulminant myocarditis with immune checkpoint inhibitor nivolumab. Can J Cardiol. 2018;34(6):812 e811-812 e813.

6. Inayat F, Masab M, Gupta S, Ullah W. New drugs and new toxicities: pembrolizumab-induced myocarditis. BMJ Case Rep. 2018;2018:bcr-2017-223252.

7. Tajmir-Riahi A, Bergmann T, Schmid M, Agaimy A, Schuler G, Heinzerling L. Life-threatening autoimmune cardiomyopathy reproducibly induced in a patient by checkpoint inhibitor therapy. J Immunother. 2018;41(1):35-38.

8. Arangalage D, Delyon J, Lermuzeaux M, Ekpe K, Ederhy S, Pages C, Lebbe C. Survival after fulminant myocarditis induced by immune-checkpoint inhibitors. Ann Intern
Med. 2017;167(9):683-684.

9. Matson DR, Accola MA, Rehrauer WM, Corliss RF. Fatal myocarditis following treatment with the PD-1 inhibitor nivolumab. J Forensic Sci. 2018;63(3):954-957.

10. Berg DD, Vaduganathan M, Nohria A, Davids MS, Alyea EP, Torre M, Padera RF, Jr. Immune-related fulminant myocarditis in a patient receiving ipilimumab therapy for relapsed chronic myelomonocytic leukaemia. Eur J Heart Fail. 2017;19(5):682-685.

11. Tay RY, Blackley E, McLean C, Moore M, Bergin P, Gill $\mathrm{S}$, Haydon A. Successful use of equine anti-thymocyte globulin (ATGAM) for fulminant myocarditis secondary to nivolumab therapy. Br J Cancer. 2017;117(7):921-924.

12. Sauer R, Kiewe P, Desole M, Schuler M, Theissig F, Roth A, Mairinger T. [Lymphocytic myocarditis in a patient with metastatic clear cell renal cell carcinoma treated with Nivolumab]. Pathologe. 2017;38(6):535-539.

13. Tadokoro T, Keshino E, Makiyama A, Sasaguri T, Ohshima K, Katano H, Mohri M. Acute lymphocytic myocarditis with anti-PD-1 antibody nivolumab. Circ Heart Fail. 2016;9(10):e003514.

14. Semper H, Muehlberg F, Schulz-Menger J, Allewelt M, Grohe C. Drug-induced myocarditis after nivolumab treatment in a patient with PDL1- negative squamous cell carcinoma of the lung. Lung Cancer. 2016;99:117-119.

15. Gibson R, Delaune J, Szady A, Markham M. Suspected autoimmune myocarditis and cardiac conduction abnormalities with nivolumab therapy for non-small cell lung cancer. BMJ Case Rep. 2016;2016:bcr2016216228.

16. Kimura T, Fukushima S, Miyashita A, Aoi J, Jinnin M, Kosaka T, Ando Y, et al. Myasthenic crisis and polymyositis induced by one dose of nivolumab. Cancer Sci. 2016;107(7):1055-1058.

17. Mehta A, Gupta A, Hannallah F, Koshy T, Reimold S. Myocarditis as an immune-related adverse event with ipilimumab/nivolumab combination therapy for metastatic melanoma. Melanoma Res. 2016;26(3):319-320.

18. Koelzer VH, Rothschild SI, Zihler D, Wicki A, Willi B, Willi N, Voegeli $M$, et al. Systemic inflammation in a melanoma patient treated with immune checkpoint inhibitors-an autopsy study. J Immunother Cancer. 2016;4:13.

19. Laubli H, Balmelli C, Bossard M, Pfister O, Glatz K, Zippelius A. Acute heart failure due to autoimmune myocarditis under pembrolizumab treatment for metastatic melanoma. J Immunother Cancer. 2015;3:11.

20. Nierstedt RT, Yeahia R, Barnett KM. Unanticipated myocarditis in a surgical patient treated with pembrolizumab: a case report. A A Pract. 2020;14(6):e01177.

21. Ansari-Gilani K, Tirumani SH, Smith DA, Nelson A, Alahmadi A, Hoimes CJ, Ramaiya NH. Myocarditis associated with immune checkpoint inhibitor therapy: a case report of three patients. Emerg Radiol. 2020;27(4):455460.

22. Lee DH, Armanious M, Huang J, Jeong D, Druta M, Fradley MG. Case of pembrolizumab-induced myocarditis presenting as torsades de pointes with safe re-challenge. J Oncol Pharm Pract. 2020;26(6):1544-1548.

23. Hardy T, Yin M, Chavez JA, Ivanov I, Chen W, Nadasdy T, Brodsky SV. Acute fatal myocarditis after a single dose 
of anti-PD-1 immunotherapy, autopsy findings: a case report. Cardiovasc Pathol. 2020;46:107202.

24. Arponen O, Skytta T. Immune checkpoint inhibitor-induced myocarditis not visible with cardiac magnetic resonance imaging but detected with PET-CT: a case report. Acta Oncol. 2020;59(4):490-492.

25. Edahiro R, Shiroyama T, Hijiki S, Nojima S, Shirai Y, Koyama S, Kumanogoh A. Severe myocarditis with slight lymphocytic infiltration after nivolumab treatment. Lung Cancer. 2020;140:116-117.

26. Valenti-Azcarate R, Esparragosa Vazquez I, Toledano Illan C, Idoate Gastearena MA, Gallego Perez-Larraya J. Nivolumab and Ipilimumab-induced myositis and myocarditis mimicking a myasthenia gravis presentation. Neuromuscul Disord. 2020;30(1):67-69.

27. Guiney TE, Lopes MS, Kalra MK, Mooradian MJ, Neilan TG, Stone JR. Case 30-2019: A 65-year-old woman with lung cancer and chest pain. N Engl J Med. 2019;381(13):1268-1277.

28. Peters N, Greally M, Breen K, Fabre A, Blazkova S. Immunotherapy- a double edged sword; a case of fatal myocarditis and complete response. Ir Med J. 2019;112(5):937.

29. Wang Q, Hu B. Successful therapy for autoimmune myocarditis with pembrolizumab treatment for nasopharyngeal carcinoma. Ann Transl Med. 2019;7(11):247.

30. Tan JL, Mugwagwa AN, Cieslik L, Joshi R. Nivolumabinduced myocarditis complicated by complete atrioventricular block in a patient with metastatic non-small cell lung cancer. BMJ Case Rep. 2019;12(7):e229963.

31. Saibil SD, Bonilla L, Majeed H, Sotov V, Hogg D, Chappell MA, Cybulsky M, et al. Fatal myocarditis and rhabdomyositis in a patient with stage IV melanoma treated with combined ipilimumab and nivolumab. Curr Oncol. 2019;26(3):e418-e421.

32. Fazel M, Jedlowski PM. Severe myositis, myocarditis, and myasthenia gravis with elevated anti-striated muscle antibody following single dose of ipilimumab-nivolumab therapy in a patient with metastatic melanoma. Case Reports Immunol. 2019;2019:2539493.

33. Gallegos C, Rottmann D, Nguyen VQ, Baldassarre LA. Myocarditis with checkpoint inhibitor immunotherapy: case report of late gadolinium enhancement on cardiac magnetic resonance with pathology correlate. Eur Heart J Case Rep. 2019;3(1):yty149.

34. Agrawal N, Khunger A, Vachhani P, Colvin TA, Hattoum A, Spangenthal E, Curtis AB, et al. Cardiac toxicity associated with immune checkpoint inhibitors: case series and review of the literature. Case Rep Oncol. 2019;12(1):260276.

35. So H, Ikeguchi R, Kobayashi M, Suzuki M, Shimizu Y, Kitagawa K. PD-1 inhibitor-associated severe myasthenia gravis with necrotizing myopathy and myocarditis. J Neurol Sci. 2019;399:97-100.

36. Monge C, Maeng H, Brofferio A, Apolo AB, Sathya B, Arai AE, Gulley JL, et al. Myocarditis in a patient treated with Nivolumab and PROSTVAC: a case report. J Immunother Cancer. 2018;6(1):150.

37. Baldetti L, Melillo F, Beneduce A, Camici PG. Combined checkpoint inhibitor-associated myocarditis and pulmo- nary vasculitis mimicking acute pulmonary embolism. Eur Heart J Cardiovasc Imaging. 2019;20(2):243.

38. Lie G, Weickhardt A, Kearney L, Lam Q, John T, Liew $\mathrm{D}$, Arulananda S. Nivolumab resulting in persistently elevated troponin levels despite clinical remission of myocarditis and myositis in a patient with malignant pleural mesothelioma: case report. Transl Lung Cancer Res. 2020;9(2):360-365.

39. von Itzstein MS, Khan S, Popat V, Lu R, Khan SA, Fattah FJ, Park JY, et al. Statin intolerance, anti-HMGCR antibodies, and immune checkpoint inhibitor-associated myositis: a "Two-Hit" autoimmune toxicity or clinical predisposition? Oncologist. 2020;25(8):e1242-e1245.

40. Shen L, Chen H, Wei Q. Immune-therapy-related toxicity events and dramatic remission after a single dose of pembrolizumab treatment in metastatic thymoma: a case report. Front Immunol. 2021;12:621858.

41. Elder CT, Davis EC, Jaipal S, Wight CE. Immune-checkpoint inhibitor toxicity during a pandemic: Overcoming patient fears to provide care. A case report. J Oncol Pharm Pract. 2021:10781552211012782.

42. Alhumaid W, Yogasundaram H, Senaratne JM. Slow bidirectional ventricular tachycardia as a manifestation of immune checkpoint inhibitor myocarditis. Eur Heart J. 2021;42(29):2868.

43. Jenkins JD, Camara-Lemarroy C, Joseph JT, Brust T. Multiple Immune-Related Adverse Event Overlap in Two Patients Treated with Pembrolizumab. Can J Neurol Sci. 2021:1-3.

44. Schiopu SRI, Kasmann L, Schonermarck U, Fischereder M, Grabmaier U, Manapov F, Rauch J, et al. Pembrolizumab-induced myocarditis in a patient with malignant mesothelioma: plasma exchange as a successful emerging therapy-case report. Transl Lung Cancer Res. 2021;10(2):1039-1046.

45. Barham W, Guo R, Park SS, Herrmann J, Dong H, Yan Y. Case report: simultaneous hyperprogression and fulminant myocarditis in a patient with advanced melanoma following treatment with immune checkpoint inhibitor therapy. Front Immunol. 2020;11:561083.

46. Xie X, Wang F, Qin Y, Lin X, Xie Z, Liu M, Ouyang M, et al. Case report: fatal multiorgan failure and Heterochronous pneumonitis following pembrolizumab treatment in a patient with large-cell neuroendocrine carcinoma of lung. Front Pharmacol. 2020;11:569466.

47. Yanase T, Moritoki Y, Kondo H, Ueyama D, Akita H, Yasui T. Myocarditis and myasthenia gravis by combined nivolumab and ipilimumab immunotherapy for renal cell carcinoma: A case report of successful management. Urol Case Rep. 2021;34:101508.

48. Fazal M, Prentice DA, Kho LK, Fysh E. Nivolumab-associated myositis myocarditis and myasthenia and anti-striated muscle antibodies. Intern Med J. 2020;50(8):10031006.

49. Sessums M, Yarrarapu S, Guru PK, Sanghavi DK. Atezolizumab-induced myositis and myocarditis in a patient with metastatic urothelial carcinoma. BMJ Case Rep. 2020;13(12):e236357.

50. Salido Iniesta M, Lopez Lopez L, Carreras Costa F, Sionis 
A. A different type of acute myocarditis: a case report of acute autoimmune myocarditis mediated by anti-PD-1 T lymphocyte receptor (pembrolizumab). Eur Heart J Case Rep. 2020;4(5):1-6.

51. Giancaterino S, Abushamat F, Duran J, Lupercio F, DeMaria A, Hsu JC. Complete heart block and subsequent sudden cardiac death from immune checkpoint inhibitor-associated myocarditis. HeartRhythm Case Rep. 2020;6(10):761-764.

52. Tanabe J, Watanabe N, Endo A, Nagami T, Inagaki S, Tanabe K. Asymptomatic immune checkpoint inhibitorassociated myocarditis. Intern Med. 2021;60(4):569-573.

53. Sakai S, Tajiri K, Li S, Ieda M. Fatal cerebral haemorrhagic infarction due to left ventricular thrombus after healing of immune checkpoint inhibitor-associated myocarditis. Eur Heart J Case Rep. 2020;4(4):1-2.

54. Tsuruda T, Yoshikawa N, Kai M, Yamaguchi M, Toida R, Kodama T, Kajihara K, et al. The cytokine expression in patients with cardiac complication after immune checkpoint inhibitor therapy. Intern Med. 2021;60(3):423-429.

55. Matsui H, Kawai T, Sato Y, Ishida J, Kadowaki H, Akiyama Y, Yamada Y, et al. A fatal case of myocarditis following myositis induced by pembrolizumab treatment for metastatic upper urinary tract urothelial carcinoma. Int Heart J. 2020;61(5):1070-1074.

56. Diamantopoulos PT, Tsatsou K, Benopoulou O, Bonou M, Anastasopoulou A, Mastrogianni E, Gogas H. Concomitant development of neurologic and cardiac immune-related adverse effects in patients treated with immune checkpoint inhibitors for melanoma. Melanoma Res. 2020;30(5):484-491.

57. Tan NYL, Anavekar NS, Wiley BM. Concomitant myopericarditis and takotsubo syndrome following immune checkpoint inhibitor therapy. BMJ Case Rep. 2020;13(9):e235265.

58. Liu Y, Jiang L. Tofacitinib for treatment in immune-mediated myocarditis: The first reported cases. J Oncol Pharm Pract. 2021;27(3):739-746.

59. Jeyakumar N, Etchegaray M, Henry J, Lelenwa L, Zhao B, Segura A, Buja LM. The terrible triad of checkpoint inhibition: a case report of myasthenia gravis, myocarditis, and myositis induced by Cemiplimab in a patient with metastatic cutaneous squamous cell carcinoma. Case Reports Immunol. 2020;2020:5126717.

60. Szuchan C, Elson L, Alley E, Leung K, Camargo AL, Elimimian E, Nahleh Z, et al. Checkpoint inhibitor-induced myocarditis and myasthenia gravis in a recurrent/ metastatic thymic carcinoma patient: a case report. Eur Heart J Case Rep. 2020;4(3):1-8.

61. Al-Obaidi A, Parker NA, Choucair K, Alderson J, Deutsch JM. A case of acute heart failure following immunotherapy for metastatic lung cancer. Cureus. 2020;12(5):e8093.

62. Khoury ZH, Hausner PF, Idzik-Starr CL, Frykenberg MRA, Brooks JK, Dyalram D, Basile JR, et al. Combination nivolumab/ipilimumab immunotherapy for melanoma with subsequent unexpected cardiac arrest: a case report and review of literature. J Immunother. 2019;42(8):313-317.

63. Martin Huertas R, Saavedra Serrano C, Perna C, Ferrer
Gomez A, Alonso Gordoa T. Cardiac toxicity of immunecheckpoint inhibitors: a clinical case of nivolumab-induced myocarditis and review of the evidence and new challenges. Cancer Manag Res. 2019;11:4541-4548.

64. Matsuo K, Ishiguro T, Najama T, Shimizu Y, Kobayashi Y, Mutou M. Nivolumab-induced myocarditis successfully treated with corticosteroid therapy: a case report and review of the literature. Intern Med. 2019;58(16):23672372 .

65. Immunotherapy for Cancer [Internet]. 2015 [cited 2021 May 30]. Available from: https://www.cancer.gov/aboutcancer/treatment/types/immunotherapy.

66. Guo L, Zhang H, Chen B. Nivolumab as Programmed Death-1 (PD-1) inhibitor for targeted immunotherapy in tumor. J Cancer. 2017;8(3):410-416.

67. Fessas P, Lee H, Ikemizu S, Janowitz T. A molecular and preclinical comparison of the PD-1-targeted T-cell checkpoint inhibitors nivolumab and pembrolizumab. Semin Oncol. 2017;44(2):136-140.

68. Paccaly AJ, Migden MR, Papadopoulos KP, Yang F, Davis JD, Rippley RK, Lowy I, et al. Fixed dose of Cemiplimab in patients with advanced malignancies based on population pharmacokinetic analysis. Adv Ther. 2021;38(5):2365-2378

69. Jiang Y, Chen M, Nie H, Yuan Y. PD-1 and PD-L1 in cancer immunotherapy: clinical implications and future considerations. Hum Vaccin Immunother. 2019;15(5):11111122.

70. Mechanism of Action [Internet]. [cited May 30, 2021]. Available from: https://www.hcp.yervoy.com/mechanism-of-action.

71. Sansom DM. CD28, CTLA-4 and their ligands: who does what and to whom? Immunology. 2000;101(2):169-177.

72. Kooshkaki O, Derakhshani A, Hosseinkhani N, Torabi M, Safaei S, Brunetti O, Racanelli V, et al. Combination of ipilimumab and nivolumab in cancers: from clinical practice to ongoing clinical trials. Int J Mol Sci. 2020;21(12):4427.

73. Zhang L, Reynolds KL, Lyon AR, Palaskas N, Neilan TG. The Evolving Immunotherapy Landscape and the Epidemiology, Diagnosis, and Management of Cardiotoxicity: JACC: CardioOncology Primer. JACC CardioOncol. 2021;3(1):35-47.

74. Johnson DB, Balko JM, Compton ML, Chalkias S, Gorham J, Xu Y, Hicks M, et al. Fulminant myocarditis with combination immune checkpoint blockade. N Engl J Med. 2016;375(18):1749-1755.

75. Tarrio ML, Grabie N, Bu DX, Sharpe AH, Lichtman AH. PD-1 protects against inflammation and myocyte damage in $\mathrm{T}$ cell-mediated myocarditis. J Immunol. 2012;188(10):4876-4884.

76. Okazaki T, Tanaka Y, Nishio R, Mitsuiye T, Mizoguchi A, Wang J, Ishida M, et al. Autoantibodies against cardiac troponin I are responsible for dilated cardiomyopathy in PD-1-deficient mice. Nat Med. 2003;9(12):1477-1483.

77. Tivol EA, Borriello F, Schweitzer AN, Lynch WP, Bluestone JA, Sharpe AH. Loss of CTLA-4 leads to massive lymphoproliferation and fatal multiorgan tissue destruction, revealing a critical negative regulatory role of 
CTLA-4. Immunity. 1995;3(5):541-547.

78. Kadowaki H, Akazawa H, Ishida J, Komuro I. Mechanisms and management of immune checkpoint inhibitorrelated cardiac adverse events. JMA J. 2021;4(2):91-98.

79. Patel RP, Parikh R, Gunturu KS, Tariq RZ, Dani SS, Ganatra S, Nohria A. Cardiotoxicity of immune checkpoint inhibitors. Curr Oncol Rep. 2021;23(7):79.

80. Al-Akchar M, Kiel J. Acute Myocarditis. [Updated May 12, 2021]. In: StatPearls [Internet]. Treasure Island (FL): StatPearls Publishing; 2021 Jan. Available from: https:// www.ncbi.nlm.nih.gov/books/NBK441847.

81. Stein-Merlob AF, Rothberg MV, Ribas A, Yang EH. Cardiotoxicities of novel cancer immunotherapies. Heart. 2021.

82. Gurdogan M, Yalta K. Myocarditis associated with immune checkpoint inhibitors: Practical considerations in diagnosis and management. Anatol J Cardiol. 2020;24(2): 68-75.

83. Awadalla M, Mahmood SS, Groarke JD, Hassan MZO, Nohria A, Rokicki A, Murphy SP, et al. Global longitudinal strain and cardiac events in patients with immune checkpoint inhibitor-related myocarditis. J Am Coll Cardiol. 2020;75(5):467-478.

84. Brahmer JR, Lacchetti C, Schneider BJ, Atkins MB, Brassil KJ, Caterino JM, Chau I, et al. Management of immune-related adverse events in patients treated with immune checkpoint inhibitor therapy: American Society of Clinical Oncology Clinical Practice Guideline. J Clin Oncol. 2018;36(17):1714-1768.

85. Wang DY, Okoye GD, Neilan TG, Johnson DB, Moslehi JJ. Cardiovascular toxicities associated with cancer immunotherapies. Curr Cardiol Rep. 2017;19(3):21. 